ESAIM: COCV 27 (2021) 3

https://doi.org/10.1051/cocv/2020083
ESAIM: Control, Optimisation and Calculus of Variations

www.esaim-cocv.org

\title{
SYMMETRY RESULTS FOR VARIATIONAL ENERGIES ON CONVEX POLYGONS
}

\author{
DORIN BuCUR ${ }^{1, *}$ AND IlARIA FrAGAL $\grave{A}^{2}$
}

\begin{abstract}
We prove that, in the class of convex polygons with a given number of sides, the regular $n$-gon is optimal for some shape optimization problems involving the torsional rigidity, the principal frequency of the Laplacian, or the logarithmic capacity.
\end{abstract}

Mathematics Subject Classification. 49Q10, 35A15, 52B60, 52A40.

Received August 27, 2020. Accepted November 27, 2020.

\section{INTRODUCTION AND MAIN RESULTS}

Investigating the symmetry of solutions to free boundary problems is a classic question in shape optimization. Several techniques have been developed towards this purpose, e.g. moving planes, rearrangements, variational methods, etc. A certain number of conjectures inspired by natural phenomena like shapes of drops, soap bubbles, circular drums have been solved. Meanwhile, there are some natural phenomena, like the bees honeycomb or the columnar jointing at Giant's Causeway where the observed structures are of polygonal type (see for instance $[5,19])$, and the question is to understand why these structures pop up. The mathematical models may be very different from one problem to another, but usually they involve some variational energies for which the equilibrium is expected to lead to these polygonal structures. A natural associated mathematical question is to understand what are the optimal shapes for those variational energies as soon as the admissible geometries is restricted, for instance, to a class of polygons with a fixed number of sides. This rigidity may simplify some aspects of the mathematical analysis, but makes the search for symmetry a very hard task.

Aim of this paper is to provide some new isoperimetrc-type inequalities for variational energies over the class of convex polygons with a given number of sides. The variational energies we consider are classical shape functionals, such as the torsional rigidity, the principal eigenvalue of the Laplacian, or the logarithmic capacity. Recall that, for every $K$ in the class $\mathcal{K}_{0}^{2}$ convex compact sets with nonempty interior, they are defined respectively by

$$
\tau(K):=-\inf _{u \in H_{0}^{1}(K)} \int_{K}\left(|\nabla u|^{2}-2 u\right) \mathrm{d} x
$$

Keywords and phrases: Shape optimization, polygons, Polya's conjecture, eigenvalues, torsional rigidity.

${ }^{1}$ Université Savoie Mont Blanc, Laboratoire de Mathématiques, CNRS UMR 5127, Campus Scientifique, 73376 Le-BourgetDu-Lac, France.

2 Dipartimento di Matematica Politecnico di Milano Piazza Leonardo da Vinci, 3220133 Milano, Italy.

* Corresponding author: dorin.bucur@univ-savoie.fr 


$$
\begin{gathered}
\lambda_{1}(K):=\inf _{u \in H_{0}^{1}(K) \backslash\{0\}} \frac{\int_{K}|\nabla u|^{2} \mathrm{~d} x}{\int_{K}|u|^{2} \mathrm{~d} x}, \\
\operatorname{cap}(K):=\exp \left(-\lim _{|x| \rightarrow+\infty}(u(x)-\log |x|)\right),
\end{gathered}
$$

where in the last definition $u$ is the log-equilibrium potential of $K$ (cf. Eq. (1.10)).

The study of isoperimetric-type inequalities for these functionals is object of a long-lasting interest: started in the mid of last century with the fundamental contribution by Pólya-Szegö [21], it is still a hot topic in modern shape optimization (see for instance the recent monograph [16]).

Among the most relevant open problems in this field, a challenging conjecture by Pólya-Szegö predicts the extremality of the regular $n$-gon, for any of the functionals above, in the class of $n$-gons with a given area. While for $n=3$ and $n=4$ the conjecture was proved by Pólya-Szegö themselves via the classical tool of Steiner symmetrization (see [21], p. 58 for $n \geq 5$ at present the unique solved case is the one of logarithmic capacity (see [24]).

Related contributions include $[4,13,20]$, and also the present work is inspired by the conjecture. In fact our aim is to shed some light on the extremality properties of the regular $n$-gon: we show that it solves some optimization problems for any of the variational energies $F$ introduced in (1.1)-(1.2)-(1.3) over the class $\mathcal{P}_{n}$ of convex polygons with $n$ sides, subject to different kinds of constraints. Precisely, the constraints we are going to consider are: (i) first variation constraint; (ii) symmetric content constraint; (iii) perimeter constraint.

\subsection{First variation constraint}

The first problem we consider consists in minimizing, over the class $\mathcal{P}_{n}$, what we call the isoperimetric quotient associated with $F$, namely the dilation invariant shape functional

$$
\mathcal{I}_{F}(K):=\left.\frac{\mathrm{d}}{\mathrm{d} t} F^{\frac{1}{\alpha}}(K+t B)\right|_{t=0} .
$$

Here $K+t B$ denotes the Minkowski addition between $K$ and the unit ball $B$, and $\alpha$ denotes the homogeneity degree of $F$ under domain dilation, which is given by

$$
\alpha= \begin{cases}4 & \text { if } F=\tau \\ -2 & \text { if } F=\lambda_{1} \\ 1 & \text { if } F=\text { cap }\end{cases}
$$

The terminology is justified by the fact that, if $F$ is replaced by the volume functional, $\mathcal{I}_{F}$ gives back $(1 / n)|K|^{\frac{1}{n}-1}|\partial K|$, namely (a multiple of) the classical isoperimetric quotient.

To make the expression of $\mathcal{I}_{F}$ more explicit let us observe that, for $F=\tau$ and $F=\lambda_{1}$ (in which cases $\alpha \neq 1$ ), we have

$$
\mathcal{I}_{F}(K)=\left.\frac{1}{\alpha} F^{\frac{1}{\alpha}-1}(K) \frac{\mathrm{d}}{\mathrm{d} t} F(K+t B)\right|_{t=0}=\frac{1}{|\alpha|} F^{\frac{1}{\alpha}-1}(K)\left|\mu_{K}\right|,
$$

while for $F=$ cap (in which case $\alpha=1$ ), we have

$$
\mathcal{I}_{F}(K)=\left.F(K) \frac{\mathrm{d}}{\mathrm{d} t} \log F(K+t B)\right|_{t=0}=\frac{1}{2 \pi} F(K)\left|\mu_{K}\right|
$$


As explained more in detail in Section 2.1 below, here $\left|\mu_{K}\right|$ represents the total mass of the first variation measure of $F$ (for $F=\tau, \lambda_{1}$ ) and of $\log F$ (for $F=$ cap). In all the cases under study, it can be written as

$$
\left|\mu_{K}\right|:=\int_{\partial K}\left|\nabla u_{K}\right|^{2} \mathrm{~d} \mathcal{H}^{1},
$$

where $u_{K}$ is the torsion function, the first Dirichlet eigenfunction of the Laplacian, or the logarithmic-potential of $K$, namely the unique solution to

$$
\begin{gathered}
-\Delta u=1 \text { in } K, \quad u=0 \text { on } \partial K, \\
-\Delta u=\lambda_{1}(K) u \text { in } K, \quad u=0 \text { on } \partial K, \quad \int_{K} u^{2} \mathrm{~d} x=1, \\
\Delta u=0 \text { in } \mathbb{R}^{2} \backslash K, \quad u=0 \text { on } \partial K, \quad u(x) \sim \log |x| \text { as }|x| \rightarrow+\infty .
\end{gathered}
$$

Thus, studying the shape optimization problem

$$
\min \left\{\mathcal{I}_{F}(P): P \in \mathcal{P}_{n}\right\},
$$

amounts to determine which polygons in the class $\mathcal{P}_{n}$, having a prescribed energy $F$, minimize the quantity $\left|\mu_{P}\right|$ in (1.8) (in other words, this amounts to determine polygons of given energy which are the "most stable" when perturbed by addition of a ball).

There are reasons to expect that the regular $n$-gon $P_{n}^{*}$ is optimal. The first one is the well-known fact that $P_{n}^{*}$ solves problem (1.12) in the geometric setting when $F$ is replaced by the volume functional. Moreover, symmetry is suggested by the fact that, if we enlarge the class of competitors in (1.12) to all convex bodies in $\mathcal{K}_{0}^{2}$, the solution is given by the ball. This has been proved in [18], Corollary 3.19 for $F=\lambda_{1}$, in [6], Equation (3.13) for $F=\tau$, and in [25], Theorems 4.1 and 4.4 for $F=$ cap, see also Remark 2.2.

Actually, we show that the following discrete version of these results holds true:

Theorem 1.1. Let $F$ be given by (1.1), (1.2), or (1.3). Then the regular n-gon $P_{n}^{*}$ solves problem (1.12).

The proof relies on a kind of Minkowski first inequality for the functionals $F$, namely an inequality which transposes to our variational setting the so-called Minkowski first inequality for volume [22], equation (7.18) (see Prop. 2.1).

More precisely, to obtain the optimality of the regular $n$-gon for problem (1.12), we proceed in two steps: in the first one we minimize $\mathcal{I}_{F}$ in the class of polygons with fixed normals, and by means of Proposition 2.1 we show that the optimal polygon is circumscribed to a disk. Then we observe that, for circumscribed polygons, it holds

$$
\mathcal{I}_{F}(P)=\frac{F^{\frac{1}{\alpha}}(P)}{\rho(P)}
$$

where $\rho(P)$ denotes the inradius of $P$. Hence we conclude by invoking a result due to Solynin which states that, under a fixed inradius, the regular $n$-gon minimizes $\tau$ and cap, while it maximizes $\lambda_{1}$ (see [23], Thm. 2).

\subsection{Symmetric content constraint}

The second problem we consider involves instead a purely geometrical constraint. Given $P \in \mathcal{P}_{n}$, we introduce the symmetric content of $P$ as the quantity

$$
\sigma(P):=\inf \left\{\frac{1}{n} \sum_{i=1}^{n} h_{P}\left(\nu_{i}\right):\left(\nu_{1}, \ldots, \nu_{n}\right) \in \mathcal{E}_{n}\left(S^{1}\right)\right\} .
$$


Here $h_{P}$ is the support function of $P$ (whose definition is recalled in Sect. 2.1), and $\mathcal{E}_{n}\left(S^{1}\right)$ denotes the class of $n$-tuples of vectors in $S^{1}$ which are equidistributed, meaning that the angle formed by $\nu_{i}$ with $\nu_{i+1}$ is independent of $i \in\{1, \ldots, n\}$ (with the convention $\nu_{n+1}=\nu_{1}$ ). To the best of our knowledge, the geometric quantity $\sigma(P)$ has not been previously considered in the literature. Some bounds for it, in terms of inradius and perimeter, are given in Section 2.1. In view of the homogeneity of degree 1 under dilations of the symmetric content, working under this constraint amounts to consider the shape optimization problem

$$
\max \left\{\frac{F(P)^{\frac{1}{\alpha}}}{\sigma(P)}: P \in \mathcal{P}_{n}\right\}
$$

Also in this case, we prove that the regular $n$-gon is optimal:

Theorem 1.2. Let $F$ be given by (1.1), (1.2), or (1.3). Then the regular n-gon $P_{n}^{*}$ solves problem (1.14).

The proof of Theorem 1.2 is somehow "dual" to that of Theorem 1.1, meaning that we apply again the variational version of Minkowski first inequality (Prop. 2.1), but with the roles of the two involved polygons interplayed. However, the analogy is not complete since in case of Theorem 1.2 we proceed in a single step ( $c f$. Rem. 3.2).

\subsection{Perimeter constraint}

Concerning the optimization problem

$$
\max \left\{\frac{F(P)^{\frac{1}{\alpha}}}{|\partial P|}: P \in \mathcal{P}_{n}\right\},
$$

the regular $n$-gon is expected to be optimal as a consequence of Pólya-Szegö conjecture. Let us recall that, limited to the case $F=$ cap, this has been established in [23], Theorem 10, but the same proof does not work for $\tau$ and $\lambda_{1}$. In this direction, we move some step forward: inspired by the case of the constraints previously analyzed, we consider problem (1.15) when the admissible class is restricted to the family of polygons with given normals, or equivalently, with given inner angles.

Firstly we consider problem (1.15) over the subclass $\widehat{\mathcal{P}_{n}}$ of polygons having equal inner angles. As a consequence of Theorem 1.2 we obtain the optimality of the regular $n$-gon:

Theorem 1.3. Let $F$ be given by (1.1), (1.2), or (1.3), and let $\widehat{\mathcal{P}}_{n}$ denote the class of equiangular polygons in $\mathcal{P}_{n}$. Then the regular $n$-gon $P_{n}^{*}$ solves

$$
\max \left\{\frac{F(P)^{\frac{1}{\alpha}}}{|\partial P|}: P \in \widehat{\mathcal{P}}_{n}\right\}
$$

Then we consider problem (1.15) over the subclass $\mathcal{P}_{n}\left(\theta_{1}, \ldots, \theta_{n}\right)$ of convex polygons having a fixed arbitrary $n$-uple $\left(\theta_{1}, \ldots, \theta_{n}\right)$ of inner angles. We prove that the solution is unique, and that it can be identified with the only polygon in the admissible class which satisfies an overdetermined boundary value problem involving a condition of integral type on each side. Theorem 1.4 below should be compared with Theorem 1.3 in [14] and Theorems 5-8-11 in [11], where the analogous overdetermined problem was considered in the extremal cases $n=3$ (triangles) and $n \rightarrow+\infty$ (arbitrary convex bodies). Note in particular that for $n=3$ the situation is quite different: indeed, since all the elements of $\mathcal{P}_{3}\left(\theta_{1}, \theta_{2}, \theta_{3}\right)$ are homothetic, the functional $F^{1 / \alpha}(P) /|\partial P|$ is constant on such class, and the overdetermined condition (1.17) is satisfied for all triangles.

Theorem 1.4. Let $F$ be given by (1.1), (1.2), or (1.3), let $n \geq 4$, and let $\mathcal{P}_{n}\left(\theta_{1}, \ldots, \theta_{n}\right)$ denote the class of polygons in $\mathcal{P}_{n}$ with inner angles given by the ordered $n$-tuple $\left(\theta_{1}, \ldots, \theta_{n}\right)$. Then there exists a unique polygon 
$\widehat{P} \in \mathcal{P}_{n}\left(\theta_{1}, \ldots, \theta_{n}\right)$ satisfying the family of overdetermined conditions

$$
\int_{S_{i}}\left|\nabla u_{\widehat{P}}\right|^{2}=\kappa\left[g\left(\theta_{i}\right)+g\left(\theta_{i+1}\right)\right] \quad \forall i=1, \ldots, n,
$$

where $g(t)=\cot (t / 2), S_{i}$ is the side adjacent to $\theta_{i}$ and $\theta_{i+1}$, and $\kappa$ is a positive constant (which is uniquely determined as $\kappa=\frac{|\alpha| F(\widehat{P})}{|\partial \widehat{P}|}$ for $F=\tau, \lambda_{1}$, and $\kappa=\frac{2 \pi}{|\partial \widehat{P}|}$ for $F=$ cap). Moreover, such polygon $\widehat{P}$ is the unique solution to

$$
\max \left\{\frac{F(P)^{\frac{1}{\alpha}}}{|\partial P|}: P \in \mathcal{P}_{n}\left(\theta_{1}, \ldots, \theta_{n}\right)\right\}
$$

Remark 1.5. We address the open problem of finding a geometric characterization of the polygon $\widehat{P}$, which could be quite helpful in order to make further progress towards the solution of problem (1.15). By now, the only property we are able to provide about $\widehat{P}$ is that, in general, it is not circumscribed around a disk; the proof of this fact is postponed to Section 3. Such piece of information is to some extent surprising, firstly by comparison with the behavior of problem (1.12), and also because we shall see below (cf. Cor. 2.5) that $\widehat{P}$ satisfies the following identities holding as well for any circumscribed polygon in $\mathcal{P}_{n}\left(\theta_{1}, \ldots, \theta_{n}\right)($ with $g(t)=\cot (t / 2))$ :

$$
\begin{aligned}
& \frac{F(P)}{|\partial P|\left|\mu_{P}\right|}=\frac{1}{|\alpha| \sum_{i=1}^{n}\left[g\left(\theta_{i}\right)+g\left(\theta_{i+1}\right)\right]} \text { if } F=\tau, \lambda_{1}, \\
& \frac{1}{|\partial P|\left|\mu_{P}\right|}=\frac{1}{2 \pi \sum_{i=1}^{n}\left[g\left(\theta_{i}\right)+g\left(\theta_{i+1}\right)\right]} \text { if } F=\text { cap . }
\end{aligned}
$$

Thanks to (1.19)-(1.20), it turns out that $\widehat{P}$ satisfies

$$
\frac{F^{1 / \alpha}(\widehat{P})}{|\partial \widehat{P}|}=\frac{\mathcal{I}_{F}(\widehat{P})}{|\alpha| \sum_{i=1}^{n}\left[g\left(\theta_{i}\right)+g\left(\theta_{i+1}\right)\right]} .
$$

Therefore, establishing the optimality of $P_{n}^{*}$ for problem (1.15) is equivalent to showing that the right hand side of the above equality is maximal at $P_{n}^{*}$.

Eventually, as a consequence of the identities (1.19)-(1.20), we derive a further extremal property of the regular $n$-gon:

Theorem 1.6. Let $F$ be given by (1.1), (1.2), or (1.3), and let $\mathcal{P}_{n}^{o}$ denote the class of circumscribed polygons in $\mathcal{P}_{n}$. Then the regular $n-$ gon $P_{n}^{*}$ solves

$$
\begin{aligned}
& \max \left\{\frac{F(P)}{|\partial P|\left|\mu_{P}\right|}: P \in \mathcal{P}_{n}^{o}\right\} \quad \text { if } F=\tau, \lambda_{1} \\
& \max \left\{\frac{1}{|\partial P|\left|\mu_{P}\right|}: P \in \mathcal{P}_{n}^{o}\right\} \quad \text { if } F=\text { cap } .
\end{aligned}
$$

The remaining of the paper is organized as follows. In Section 2, we provide some preliminary results, which concern: the Minkowski first inequality for variational functionals (Sect. 2.1), a representation formula for perimeter and its consequences (Sect. 2.2), and some properties of the symmetric content (Sect. 2.3). The proofs of Theorems 1.1 to 1.6 are then given in Section 3. 


\section{Preliminary Results}

\subsection{Minkowski first inequality for variational functionals}

We start by recalling some properties that any of the functionals $F$ introduced in (1.1)-(1.2)-(1.3) enjoys on the class $\mathcal{K}_{0}^{2}$ of convex bodies with nonempty interior.

- Brunn-Minkowski inequality.

For any $K, L \in \mathcal{K}_{0}^{2}$, and $t \in[0,1]$, setting $(1-t) K+t L=\{(1-t) x+t y: x \in K, y \in L\}$, it holds

$$
F^{\frac{1}{\alpha}}((1-t) K+t L) \geq(1-t) F^{\frac{1}{\alpha}}(K)+t F^{\frac{1}{\alpha}}(L)
$$

with equality if and only if $K$ and $L$ are homothetic. For a proof, see $[2,8]$ when $F=\tau,[3,8]$ when $F=\lambda_{1}$, and $[1,9]$ when $F=$ cap).

- Integral representation of $F$ and Hadamard variational formula.

We call first variation measure of $F$ (for $\left.F=\tau, \lambda_{1}\right)$ and of $\log F$ (for $F=$ cap) the measure $\mu_{K}$ defined on $S^{1}$ by

$$
\mu_{K}:=\left(\nu_{K}\right)_{\sharp}\left(\left|\nabla u_{K}\right|^{2} \mathcal{H}^{1}\llcorner\partial K) .\right.
$$

Here $\nu_{K}$ is the unit outer normal to $K$, which is defined $\mathcal{H}^{1}$-a.e. on $\partial K$, and $u_{K}$ denotes the unique solution respectively to problem (1.9)-(1.10)-(1.11), where the PDE has to be understood in the interior of $K$. (The meaning of the push-forward symbol $\left(\nu_{K}\right)_{\sharp}$ is that, for every $\left.\varphi \in \mathcal{C}_{0}\left(\mathbb{S}^{n-1}\right), \int_{S^{1}} \varphi d \mu_{K}=\int_{\partial K} \varphi \circ \nu_{K}\left|\nabla u_{K}\right|^{2} \mathrm{~d} \mathcal{H}^{1}\right)$.

In particular, when $K$ is a polygon with sides $S_{i}$ and normals $\nu_{i}$, we have

$$
\mu_{K}=\sum_{i=1}^{n}\left(\int_{S_{i}}\left|\nabla u_{K}\right|^{2} \mathrm{~d} \mathcal{H}^{1}\right) \delta_{\nu_{i}}
$$

Note that the total mass of $\mu_{K}$ agrees with the quantity introduced in (1.8):

$$
\left|\mu_{K}\right|:=\mu_{K}\left(S^{1}\right)=\int_{S^{1}} 1 d \mu_{K}=\int_{\partial K}\left|\nabla u_{K}\right|^{2} \mathrm{~d} \mathcal{H}^{1}
$$

The reason of the terminology adopted above for the measure $\mu_{K}$ relies in the following integral representation formulae, holding on $\mathcal{K}_{0}^{2}$ for the functionals $F$. We adopt the standard notation to indicate the support function of $K$, defined by

$$
h_{K}(\nu):=\sup _{x \in K}(x \cdot \nu) \quad \forall \nu \in \mathbb{S}^{1} .
$$

In case of $F=\tau, \lambda_{1}$, we have:

$$
\begin{aligned}
& F(K)=\frac{1}{|\alpha|} \int_{\partial K} h_{K}\left(\nu_{K}(x)\right)\left|\nabla u_{K}\right|^{2}(x) \mathrm{d} \mathcal{H}^{1}(x)=\frac{1}{|\alpha|} \int_{S^{1}} h_{K} d \mu_{K} \\
& \left.\frac{\mathrm{d}}{\mathrm{d} t} F((1-t) K+t L)\right|_{t=0^{+}}=(\operatorname{sign} \alpha)\left(\int_{S^{1}} h_{L} d \mu_{K}-\int_{S^{1}} h_{K} d \mu_{K}\right)
\end{aligned}
$$

(for a proof see [10] when $F=\tau$ and [17] when $F=\lambda_{1}$ ). 
In case of $F=$ cap, we have:

$$
\begin{gathered}
1=\frac{1}{2 \pi} \int_{\partial K} h_{K}\left(\nu_{K}(x)\right)\left|\nabla u_{K}\right|^{2}(x) d \mathcal{H}^{1}(x)=\frac{1}{2 \pi} \int_{S^{1}} h_{K} d \mu_{K} \\
\left.\frac{\mathrm{d}}{\mathrm{d} t} \log F((1-t) K+t L)\right|_{t=0^{+}}=\frac{1}{2 \pi}\left(\int_{S^{1}} h_{L} d \mu_{K}-\int_{S^{1}} h_{K} d \mu_{K}\right)
\end{gathered}
$$

(for a proof see [25]).

We can now state the variational form of Minkowski first inequality. After the proof we outline its usefulness to derive isoperimetric inequalities over $\mathcal{K}_{0}^{2}$ (see Rem. 2.2), while its application in the polygonal setting will appear in Section 3 (see Rems. 3.1, 3.2 and 3.3).

Proposition 2.1. Let $K, L \in \mathcal{K}_{0}^{2}$. We have

$$
\begin{cases}\int_{S^{1}} h_{L} d \mu_{K} \geq|\alpha| F^{1-\frac{1}{\alpha}}(K) F^{\frac{1}{\alpha}}(L) & \text { for } F=\tau, \lambda_{1} \\ \int_{S^{1}} h_{L} d \mu_{K} \geq 2 \pi F^{-1}(K) F(L) & \text { for } F=\text { cap } .\end{cases}
$$

The equality sign holds in the above inequalities if and only if $K$ and $L$ are homothetic.

Proof. By the Brunn-Minkowski inequality (2.1), the map

$$
t \mapsto \Phi(t):=F^{\frac{1}{\alpha}}((1-t) K+t L)-(1-t) F^{\frac{1}{\alpha}}(K)-t F^{\frac{1}{\alpha}}(L)
$$

is concave on $[0,1]$, and satisfies $\Phi(0)=\Phi(1)=0$. Hence its right derivative at $t=0$ (which exists by $(2.5)$ and (2.7)) satisfies

$$
\Phi_{+}^{\prime}(0) \geq 0,
$$

with equality if and only if $\Phi$ is identically 0 (which implies equality in (2.1)).

In case $F=\tau, \lambda_{1}$, by using (2.4)-(2.5), we obtain

$$
\begin{aligned}
\Phi_{+}^{\prime}(0) & =\frac{\operatorname{sign} \alpha}{\alpha} F^{\frac{1}{\alpha}-1}(K)\left(\int_{S^{1}} h_{L} d \mu_{K}-\int_{S^{1}} h_{K} d \mu_{K}\right)+F^{\frac{1}{\alpha}}(K)-F^{\frac{1}{\alpha}}(L) \\
& =\frac{1}{|\alpha|} F^{\frac{1}{\alpha}-1}(K) \int_{S^{1}} h_{L} d \mu_{K}-F^{\frac{1}{\alpha}}(L) .
\end{aligned}
$$

Inserting the above equality into (2.8), the result follows.

In case $F=$ cap, by using (2.6)-(2.7), we obtain

$$
\begin{aligned}
\Phi_{+}^{\prime}(0) & =\left.F(K) \frac{\mathrm{d}}{\mathrm{d} t} \log F((1-t) K+t L)\right|_{t=0^{+}}+F(K)-F(L) \\
& =\frac{1}{2 \pi} F(K)\left(\int_{S^{1}} h_{L} d \mu_{K}-\int_{S^{1}} h_{K} d \mu_{K}\right)+F(K)-F(L) \\
& =\frac{1}{2 \pi} F(K) \int_{S^{1}} h_{L} d \mu_{K}-F(L) .
\end{aligned}
$$


Again, inserting the above equality into (2.8), the result follows.

Remark 2.2. (i) By applying Proposition 2.1 with $K$ equal to arbitrary body in $\mathcal{K}_{0}^{2}$, and $L$ equal to the unit ball $B$, one obtains that balls solve the optimization problem (1.4) in which the class of competitors is enlarged to $\mathcal{K}_{0}^{2}$. Indeed, let us consider just to fix the ideas the case when $F=\tau, \lambda_{1}$. Since $\int h_{B} d \mu_{K}=\left|\mu_{K}\right|$, Proposition 2.1 gives $\left|\mu_{K}\right| \geq|\alpha| F^{1-\frac{1}{\alpha}}(K) F^{\frac{1}{\alpha}}(B)$, or equivalently

$$
\mathcal{I}_{F}(K)=\frac{1}{|\alpha|} F^{\frac{1}{\alpha}-1}(K)\left|\mu_{K}\right| \geq F^{\frac{1}{\alpha}}(B)=\mathcal{I}_{F}(B) \quad \forall K \in \mathcal{K}_{0}^{2} .
$$

(ii) In a dual way, by applying Proposition 2.1 with $L$ equal to arbitrary body in $\mathcal{K}_{0}^{2}$, and $K$ equal to the unit ball $B$, one obtains that balls solve the optimization problem

$$
\max \left\{\frac{F(K)^{\frac{1}{\alpha}}}{|\partial K|}: K \in \mathcal{K}_{0}^{2}\right\}
$$

Indeed (still arguing for $F=\tau, \lambda_{1}$, the case $F=$ cap being analogous), we have $d \mu_{B}=c d \theta$, where it is easily seen through formula (2.4) that $c=|\alpha| \frac{F(B)}{|\partial B|}$. Thus, Proposition 2.1 gives $|\alpha| \frac{F(B)}{|\partial B|}|\partial L| \geq|\alpha| F^{\frac{1}{\alpha}}(L) F^{1-\frac{1}{\alpha}}(B)$, or equivalently

$$
\frac{F^{\frac{1}{\alpha}}(L)}{|\partial L|} \leq \frac{F^{\frac{1}{\alpha}}(B)}{|\partial B|} \quad \forall L \in \mathcal{K}_{0}^{2}
$$

\subsection{A representation formula for the perimeter of convex polygons}

We recall that $\mathcal{P}_{n}\left(\theta_{1}, \ldots, \theta_{n}\right)$ denotes the class of polygons in $\mathcal{P}_{n}$ with inner angles given by the ordered $n$-tuple $\left(\theta_{1}, \ldots, \theta_{n}\right)$; moreover, for every $P \in \mathcal{P}_{n}\left(\theta_{1}, \ldots, \theta_{n}\right)$, we let $\nu_{i}$ be the unit outer normal to the side adjacent to $\theta_{i}$ and $\theta_{i+1}$.

Proposition 2.3. For every $P \in \mathcal{P}_{n}\left(\theta_{1}, \ldots, \theta_{n}\right)$, it holds

$$
|\partial P|=\sum_{i=1}^{n} h_{P}\left(\nu_{i}\right)\left[g\left(\theta_{i}\right)+g\left(\theta_{i+1}\right)\right] .
$$

Proof. For every fixed side $S$ of $P$ and every real parameter $\varepsilon$ sufficiently small, let us denote by $P_{\varepsilon}(S)$ the perturbed polygon constructed in the following way: denoting by $S^{\prime}$ and $S^{\prime \prime}$ the two sides of $\partial P$ adjacent to $S$, we replace the three sides $\left(S, S^{\prime}, S^{\prime \prime}\right)$ by the new sides $\left(S_{\varepsilon}, S_{\varepsilon}^{\prime}, S_{\varepsilon}^{\prime \prime}\right)$ defined as follows

- $S_{\varepsilon}$ lies on the straight-line parallel to $S$ having signed distance $\varepsilon h_{P}\left(\nu_{S}\right)$ from $S$ (where $\nu_{S}$ stands for the unit outer normal to $\partial P$ on $S$ );

- $S_{\varepsilon}^{\prime}$ and $S_{\varepsilon}^{\prime \prime}$ lie on the same straight-line containing respectively $S^{\prime}$ and $S^{\prime \prime}$;

- the lengths of $S_{\varepsilon}, S_{\varepsilon}^{\prime}$ and $S_{\varepsilon}^{\prime \prime}$ are chosen so that the three sides are consecutive (namely $\left(S_{\varepsilon}, S_{\varepsilon}^{\prime}\right)$ and $\left(S_{\varepsilon}, S_{\varepsilon}^{\prime \prime}\right)$ have one point in common).

Then we set

$$
P_{\varepsilon}:=\bigcup_{S} P_{\varepsilon}(S)
$$




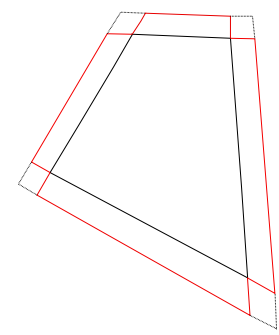

Figure 1. Construction of the polygon $P_{\varepsilon}$ in the proof of Proposition 2.3.

where the union is taken over the family of sides of $P$. Note that $P_{\varepsilon}$ is a non-convex polygon, with $n+2 n$ sides, as illustrated in Figure 1 in case of a quadrilateral.

It follows from elementary geometric arguments that

$$
\left|\partial P_{\varepsilon}\right|=|\partial P|+\varepsilon \sum_{i=1}^{n} h_{P}\left(\nu_{i}\right)\left[g\left(\theta_{i}\right)+g\left(\theta_{i+1}\right)\right] .
$$

On the other hand, by construction, the polygon $P_{\varepsilon}$ has the same perimeter as its convex hull $\operatorname{conv}\left(P_{\varepsilon}\right)$, which agrees with the homothetic polygon $(1+\varepsilon) P$. Hence,

$$
\left|\partial P_{\varepsilon}\right|=|\partial P|(1+\varepsilon) .
$$

Then (2.12) follows by combining the last two equalities.

Corollary 2.4. For every $P \in \mathcal{P}_{n}\left(\theta_{1}, \ldots, \theta_{n}\right)$, it holds

$$
\sum_{i=1}^{n}\left[g\left(\theta_{i}\right)+g\left(\theta_{i+1}\right)\right] \nu_{i}=0
$$

Proof. By the invariance of perimeter under translations and the representation formula (2.12) it follows that, for every $v \in \mathbb{R}^{n}$,

$$
|\partial P|=\sum_{i=1}^{n} h_{P}\left(\nu_{i}\right)\left[g\left(\theta_{i}\right)+g\left(\theta_{i+1}\right)\right]=\sum_{i=1}^{n} h_{P+v}\left(\nu_{i}\right)\left[g\left(\theta_{i}\right)+g\left(\theta_{i+1}\right)\right]=|\partial(P+v)|,
$$

Taking into account that

$$
h_{P+v}\left(\nu_{i}\right)=h_{P}\left(\nu_{i}\right)+v \cdot \nu_{i},
$$

we infer that

$$
v \cdot \sum_{i=1}^{n}\left[g\left(\theta_{i}\right)+g\left(\theta_{i+1}\right)\right] \nu_{i}=0
$$

and the statement follows from the arbitrariety of $v \in \mathbb{R}^{n}$. 


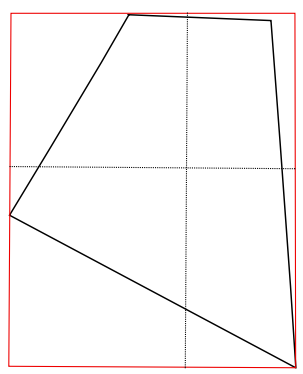

Figure 2. Construction of the polygon $\widetilde{P}$ in the proof of Proposition 2.6.

Corollary 2.5. The identities (1.19)-(1.20) hold true both for the polygon $\widehat{P}$ given by Theorem 1.3 and for the polygon in $\mathcal{P}_{n}\left(\theta_{1}, \ldots, \theta_{n}\right)$ which is circumscribed around a disk.

Proof. For the polygon $\widehat{P}$ given by Theorem 1.3, the identities (1.19)-(1.20) are obtained by summing the equalities (1.17) (for $i=1, \ldots, n$ ), and recalling the value of the constant $\kappa$ appearing therein. For the circumscribed polygon in $\mathcal{P}_{n}\left(\theta_{1}, \ldots, \theta_{n}\right)$, one has to use the representation formula (2.12) for perimeter, and the representation formula for $F$ given in (2.4)-(2.6): the required identities follow by taking into account that the support function is equal to the inradius in each of the normal directions $\nu_{i}$ (for $i=1, \ldots, n$ ).

\subsection{Some properties of the symmetric content}

Proposition 2.6. For every $P \in \mathcal{P}_{n}$ it holds

$$
\begin{aligned}
& \sigma(P) \geq \rho(P), \quad \text { with equality if } P=P_{n}^{*} ; \\
& \frac{\sigma(P)}{\sigma\left(P_{n}^{*}\right)} \geq \frac{|\partial P|}{\left|\partial P_{n}^{*}\right|} .
\end{aligned}
$$

Remark 2.7. By inequality (2.15), Theorem 1.2 is weaker than the optimality of $P_{n}^{*}$ for problem (1.15).

Proof. In view of the definition of $\sigma(P)$, inequality (2.14) follows from the lower bound $h_{P}(\nu) \geq \rho(P)$ holding for every $\nu \in S^{1}$. If $P=P_{n}^{*}$, the family $\left(\nu_{1}^{*}, \ldots, \nu_{n}^{*}\right)$ of its outer normals belongs to $\mathcal{E}_{n}\left(S^{1}\right)$. So, by choosing $\left(\nu_{1}, \ldots, \nu_{n}\right)=\left(\nu_{1}^{*}, \ldots, \nu_{n}^{*}\right)$ in the definition of $\sigma\left(P_{n}^{*}\right)$, we obtain the converse inequality $\sigma\left(P_{n}^{*}\right) \leq \rho\left(P_{n}^{*}\right)$.

To show (2.15), we argue by contradiction. Assume that the opposite inequality holds. Then one could find $\left(\nu_{1}, \ldots, \nu_{n}\right) \in \mathcal{E}_{n}\left(S^{1}\right)$ such that

$$
\frac{1}{n \rho\left(P_{n}^{*}\right)} \sum_{i=1}^{n} h_{P}\left(\nu_{i}\right) \leq \frac{|\partial P|}{\left|\partial P_{n}^{*}\right|}
$$

Since $\left|\partial P_{n}^{*}\right|=\rho\left(P_{n}^{*}\right) 2 n \cot \left(\frac{\theta_{n}}{2}\right)$, with $\theta_{n}:=\frac{(n-2) \pi}{n}$, one is led to

$$
\sum_{i=1}^{n} 2 h_{P}\left(\nu_{i}\right) \cot \left(\frac{\theta_{n}}{2}\right) \leq|\partial P| .
$$

But, by Proposition 2.3, the left hand side of the above inequality is equal to the perimeter of a convex polygon $\widetilde{P}$ having all the inner angles equal to $\theta_{n}=\frac{(n-2) \pi}{n}$ and containing $P$, as illustrated in Figure 2 in case of a quadrilateral.

Then the above inequality contradicts the monotonicity of perimeter on convex polygons. 


\section{Proof of the Results in Section 1}

\subsection{Proof of Theorem 1.1}

We divide the proof in two steps.

Step 1. Let $P \in \mathcal{P}_{n}$, and let $P_{0} \in \mathcal{P}_{n}$ denote the polygon which is circumscribed around the incircle of $P$ and has the same normals as $P$. Then it holds $\mathcal{I}_{F}(P) \geq \mathcal{I}_{F}\left(P_{0}\right)$.

We are going to apply Proposition 2.1, with $K=P$ and $L=P_{0}$.

In case $F$ equals $\tau$ or $\lambda_{1}$, we obtain

$$
\int_{S^{1}} h_{P_{0}} d \mu_{P} \geq|\alpha| F^{1-\frac{1}{\alpha}}(P) F^{\frac{1}{\alpha}}\left(P_{0}\right) .
$$

We observe that the measure $\mu_{P}$ is concentrated on the family of $n$ normal directions to the boundary of $P$ (cf. (2.3)), and in any of these directions, by the definition of $P_{0}$, the value of the support function $h_{P_{0}}$ is equal to the inradius of $P_{0}$ (which also agrees with the inradius of $P$ ). Therefore, denoting such inradius by $\rho\left(P_{0}\right)$, we have

$$
\int_{S^{1}} h_{P_{0}} d \mu_{P}=\rho\left(P_{0}\right)\left|\mu_{P}\right|
$$

Inserting (3.2) into (3.1), and recalling (1.6), we obtain

$$
\mathcal{I}_{F}(P)=\frac{1}{|\alpha|}\left|\mu_{P}\right| F^{\frac{1}{\alpha}-1}(P) \geq \frac{1}{\rho\left(P_{0}\right)} F^{\frac{1}{\alpha}}\left(P_{0}\right) .
$$

The proof of Step 1 is concluded by observing that, since $P_{0}$ is circumscribed, the r.h.s. of the above inequality agrees with $\mathcal{I}_{F}\left(P_{0}\right)$. Indeed, this is easily checked by using (1.6) and (2.4):

$$
\begin{aligned}
& \mathcal{I}_{F}\left(P_{0}\right)=\frac{1}{|\alpha|}\left|\mu_{P_{0}}\right| F\left(P_{0}\right)^{\frac{1}{\alpha}-1}=\frac{1}{|\alpha|}\left|\mu_{P_{0}}\right|\left(\frac{1}{|\alpha|} \int_{S^{1}} h_{P_{0}} d \mu_{P_{0}}\right)^{\frac{1}{\alpha}-1}=\left(\frac{1}{|\alpha|}\right)^{\frac{1}{\alpha}}\left|\mu_{P_{0}}\right|^{\frac{1}{\alpha}} \rho\left(P_{0}\right)^{\frac{1}{\alpha}-1}, \\
& \frac{1}{\rho\left(P_{0}\right)} F^{\frac{1}{\alpha}}\left(P_{0}\right)=\frac{1}{\rho\left(P_{0}\right)}\left(\frac{1}{|\alpha|} \int_{S^{1}} h_{P_{0}} d \mu_{P_{0}}\right)^{\frac{1}{\alpha}}=\left(\frac{1}{|\alpha|}\right)^{\frac{1}{\alpha}}\left|\mu_{P_{0}}\right|^{\frac{1}{\alpha}} \rho\left(P_{0}\right)^{\frac{1}{\alpha}-1} .
\end{aligned}
$$

In case $F$ equals cap, we argue in a similar way. By applying Proposition 2.1, still with $K=P$ and $L=P_{0}$, we obtain

$$
\int_{S^{1}} h_{P_{0}} d \mu_{P} \geq 2 \pi F^{-1}(P) F\left(P_{0}\right)
$$

Inserting (3.2) into (3.3), and recalling (1.7), we obtain

$$
\mathcal{I}_{F}(P)=\frac{1}{2 \pi}\left|\mu_{P}\right| F(P) \geq \frac{1}{\rho\left(P_{0}\right)} F\left(P_{0}\right) .
$$

Again, we conclude by observing that, since $P_{0}$ is circumscribed, the r.h.s. of the above inequality agrees with $\mathcal{I}_{F}\left(P_{0}\right)$. Indeed,

$$
\mathcal{I}_{F}\left(P_{0}\right)=\frac{1}{2 \pi}\left|\mu_{P_{0}}\right| F\left(P_{0}\right)=\frac{1}{\rho\left(P_{0}\right)} F\left(P_{0}\right)
$$


where the first equality holds by (1.7) and the second one follows from (2.6).

Step 2. Let $P_{0} \in \mathcal{P}_{n}$ be a circumscribed polygon, and let $P_{n}^{*}$ be a regular $n$-gon. Then it holds $\mathcal{I}_{F}\left(P_{0}\right) \geq$ $\mathcal{I}_{F}\left(P_{*}\right)$.

As shown above, since $P_{0}$ is circumscribed, in all the cases under study it holds

$$
\mathcal{I}_{F}\left(P_{0}\right)=\frac{1}{\rho\left(P_{0}\right)} F^{\frac{1}{\alpha}}\left(P_{0}\right) .
$$

Clearly the same equality holds for $P_{n}^{*}$, since $P_{n}^{*}$ as well is circumscribed. Then the claim in Step 2 follows from [23], Theorem 2 .

Remark 3.1. We point out that applying Proposition 2.1 directly with $K=P$ and $L=P_{n}^{*}$ does not allow to prove Theorem 1.1, but leads merely to the following inequalities

$$
\begin{cases}\int_{S^{1}} h_{P_{n}^{*}} d \mu_{P}=\sum_{i=1}^{n} \gamma_{i} h_{P_{n}^{*}}\left(\nu_{i}\right) \geq|\alpha| F^{1-\frac{1}{\alpha}}(P) F^{\frac{1}{\alpha}}\left(P_{n}^{*}\right) & \text { for } F=\tau, \lambda_{1} \\ \int_{S^{1}} h_{P_{n}^{*}} d \mu_{P}=\sum_{i=1}^{n} \gamma_{i} h_{P_{n}^{*}}\left(\nu_{i}\right) \geq 2 \pi F^{-1}(P) F\left(P_{n}^{*}\right) & \text { for } F=\text { cap },\end{cases}
$$

where we have written $\mu_{P}=\sum_{i=1}^{n} \gamma_{i} \delta_{\nu_{i}}$, being $\nu_{i}$ the normals to the sides $S_{i}$ of $P$ and $\gamma_{i}=\int_{S_{i}}\left|\nabla u_{P}\right|^{2} \mathrm{~d} \mathcal{H}^{1}$.

\subsection{Proof of Theorem 1.2}

Let $F$ equal $\tau$ or $\lambda_{1}$. By Proposition 2.1, applied with $L=P$ and $K=P_{n}^{*}$, we infer

$$
\int_{S^{1}} h_{P} d \mu_{P_{n}^{*}} \geq|\alpha| F^{\frac{1}{\alpha}}(P) F^{1-\frac{1}{\alpha}}\left(P_{n}^{*}\right) .
$$

Next we observe that, by the symmetry of $P_{n}^{*}$, we can write

$$
\mu_{P_{n}^{*}}=\gamma\left(P_{n}^{*}\right) \sum_{i=1}^{n} \delta_{\nu_{i}^{*}},
$$

where $\left(\nu_{1}^{*}, \ldots, \nu_{n}^{*}\right)$ are the outer normals to $P_{n}^{*}$ and $\gamma\left(P_{n}^{*}\right)$ is a positive coefficient (equal to the integral over an arbitrary side of $P_{n}^{*}$ of $\left.\left|\nabla u_{P_{n}^{*}}\right|^{2}\right)$.

By combining (3.6) with the relation (2.4) written for $P_{n}^{*}$, i.e.,

$$
F\left(P_{n}^{*}\right)=\frac{1}{|\alpha|} \int_{S^{1}} h_{P_{n}^{*}} d \mu_{P_{n}^{*}}
$$

we see that

$$
\gamma\left(P_{n}^{*}\right)=\frac{|\alpha| F\left(P_{n}^{*}\right)}{n \rho\left(P_{n}^{*}\right)}
$$


In view of (3.6)-(3.7), the inequality (3.5) can be rewritten as

$$
\frac{|\alpha| F\left(P_{n}^{*}\right)}{n \rho\left(P_{n}^{*}\right)} \sum_{i=1}^{n} h_{P}\left(\nu_{i}^{*}\right) \geq|\alpha| F^{\frac{1}{\alpha}}(P) F^{1-\frac{1}{\alpha}}\left(P_{n}^{*}\right),
$$

or

$$
\frac{F^{\frac{1}{\alpha}}(P)}{\frac{1}{n} \sum_{i=1}^{n} h_{P}\left(\nu_{i}^{*}\right)} \leq \frac{F^{\frac{1}{\alpha}}\left(P_{n}^{*}\right)}{\rho\left(P_{n}^{*}\right)} .
$$

Since the outer normals $\left(\nu_{1}^{*}, \ldots, \nu_{n}^{*}\right)$ are an arbitrary family of equidistributed unit vectors, by passing to the supremum over $\left(\nu_{1}^{*}, \ldots, \nu_{n}^{*}\right) \in \mathcal{E}_{n}\left(S^{1}\right)$ in the above inequality, and recalling the definition of $\sigma(P)$, we obtain

$$
\frac{F^{\frac{1}{\alpha}}(P)}{\sigma(P)} \leq \frac{F^{\frac{1}{\alpha}}\left(P_{n}^{*}\right)}{\rho\left(P_{n}^{*}\right)} .
$$

To conclude it is enough to observe that $\rho\left(P_{n}^{*}\right)=\sigma\left(P_{n}^{*}\right)$ ( $c f$. Prop. 2.6).

In case $F$ equals cap, we proceed in a similar way. By Proposition 2.1, still applied with $L=P$ and $K=P_{n}^{*}$, we infer this time

$$
\int_{S^{1}} h_{P} d \mu_{P_{n}^{*}} \geq 2 \pi F(P) F^{-1}\left(P_{n}^{*}\right)
$$

By writing $\mu_{P_{n}^{*}}$ as in (3.6), and exploiting (2.6), we obtain that the value of $\gamma\left(P_{n}^{*}\right)$ is given by

$$
\gamma\left(P_{n}^{*}\right)=\frac{2 \pi}{n \rho\left(P_{n}^{*}\right)}
$$

so that the inequality (3.8) can be rewritten as

$$
\frac{2 \pi}{n \rho\left(P_{n}^{*}\right)} \sum_{i=1}^{n} h_{P}\left(\nu_{i}^{*}\right) \geq 2 \pi F(P) F^{-1}\left(P_{n}^{*}\right),
$$

or

$$
\frac{F(P)}{\frac{1}{n} \sum_{i=1}^{n} h_{P}\left(\nu_{i}^{*}\right)} \leq \frac{F\left(P_{n}^{*}\right)}{\rho\left(P_{n}^{*}\right)}
$$

From this point on, the proof proceeds unaltered as in the cases treated above.

Remark 3.2. Since Theorem 1.2 is obtained by applying Proposition 2.1 directly with $K=P_{n}^{*}$ and $L=P$, it can be regarded as a dual counterpart to the inequalities (3.4), rather than of Theorem 1.1. 


\subsection{Proof of Theorem 1.3}

Let $F$ be any of the three functionals $\tau, \lambda_{1}$, cap under study, and let $P \in \widehat{\mathcal{P}}_{n}$. Since $P$ is equiangular, we can choose a regular polygon $P_{n}^{*}$ having the same normals $\left(\nu_{1}^{*}, \ldots, \nu_{n}^{*}\right)$ as $P$. Following the same proof of Theorem 1.2 , we arrive at the inequality

$$
\frac{F^{\frac{1}{\alpha}}(P)}{\frac{1}{n} \sum_{i=1}^{n} h_{P}\left(\nu_{i}^{*}\right)} \leq \frac{F^{\frac{1}{\alpha}}\left(P_{n}^{*}\right)}{\rho\left(P_{n}^{*}\right)} .
$$

Now, since by assumption $P$ is equiangular and has normals $\left(\nu_{1}^{*}, \ldots, \nu_{n}^{*}\right)$, the representation formula $(2.12)$ in Proposition 2.3 gives

$$
|\partial P|=2 g\left(\theta_{n}\right) \sum_{i=1}^{n} h_{P}\left(\nu_{i}^{*}\right), \quad \text { with } \theta_{n}=\frac{(n-2) \pi}{n} .
$$

Inserting (3.11) into (3.10) we obtain

$$
\frac{F^{\frac{1}{\alpha}}(P)}{|\partial P|} \leq \frac{F^{\frac{1}{\alpha}}\left(P_{n}^{*}\right)}{2 n g\left(\theta_{n}\right) \rho\left(P_{n}^{*}\right)}=\frac{F^{\frac{1}{\alpha}}\left(P_{n}^{*}\right)}{\left|\partial P_{n}^{*}\right|}
$$

Remark 3.3. Since Theorem 1.3 deals with $n$-gons having the same normals as the regular $n$-gon, it can be read as a dual counterpart of Step 1 in the proof of Theorem 1.1. The missing issue to obtain the optimality of the regular $n$-gon in problem (1.15) is understanding a dual counterpart to Step 2 in the proof of the same theorem.

\subsection{Proof of Theorem 1.4}

Let us first remark that, denoting by $\nu_{i}$ the unit outer normal to $S_{i}$, the unique possible value for the constant $\kappa$ is easily found by multiplying both sides of (1.17) by $h_{\widehat{P}}\left(\nu_{i}\right)$, summing over $i=1, \ldots, n$, and exploiting formulas (2.4)-(2.6) and (2.12).

Next we observe that the system of equalities (1.17) is equivalent to ask that the measure $\mu_{\widehat{P}}$ agrees with the measure

$$
\lambda:=\kappa \sum_{i=1}^{n}\left[g\left(\theta_{i}\right)+g\left(\theta_{i+1}\right] \delta_{\nu_{i}},\right.
$$

where $\delta_{\nu_{i}}$ is a Dirac mass concentrated at $\nu_{i}$. Now, searching for a solution $\widehat{P}$ to the equation $\mu_{\widehat{P}}=\lambda$ amounts to prescribe the first variation measure of $F$, namely corresponds to the so-called Minkowski problem for $F$. The results proved respectively in [18] (for $F=\lambda_{1}$, cap) and in [10] (for $F=\tau$ ) ensure that there exists a unique solution $\widehat{P}$, provided the measure $\lambda$ is not concentrated on an equator of $S^{n-1}$ and is balanced. Both these conditions are readily satisfied. Indeed, the condition that $\lambda$ is not concentrated on an equator of $S^{n-1}$ is satisfied because $\nu_{i}$ are the unit outer normals to any polygon in $\mathcal{P}_{n}\left(\theta_{1}, \ldots \theta_{n}\right)$, while the condition that $\lambda$ is balanced is satisfied by Corollary 2.4. Hence there exists a unique polygon $\widehat{P}$ satisfying the overdetermined conditions (1.17).

Next, the fact that $\widehat{P}$ is a maximizer over $\mathcal{P}_{n}\left(\theta_{1}, \ldots \theta_{n}\right)$ of the quotient between $F^{1 / \alpha}$ and perimeter readily follows by applying Proposition 2.1 , with $K=\widehat{P}$ and $L$ equal to an arbitrary polygon in $\mathcal{P}_{n}\left(\theta_{1}, \ldots \theta_{n}\right)$. 
Finally, the uniqueness of a maximizer follows from Brunn-Minkowski inequality: if $\widehat{P}_{1}$ and $\widehat{P}_{2}$ were two distinct maximizers, not homothetic, one would have

$$
\frac{F^{1 / \alpha}\left(\frac{\widehat{P}_{1}+\widehat{P}_{2}}{2}\right)}{\left|\partial\left(\frac{\widehat{P}_{1}+\widehat{P}_{2}}{2}\right)\right|}>\frac{F^{1 / \alpha}\left(\widehat{P}_{1}\right)+F^{1 / \alpha}\left(\widehat{P}_{2}\right)}{\left|\partial \widehat{P}_{1}\right|+\left|\partial \widehat{P}_{2}\right|} \geq \min _{i=1,2} \frac{F^{1 / \alpha}\left(\widehat{P}_{i}\right)}{\left|\partial \widehat{P}_{i}\right|}
$$

yielding a contradiction since $\frac{\widehat{P}_{1}+\widehat{P}_{2}}{2}$ belongs to the admissible class $\mathcal{P}_{n}\left(\theta_{1}, \ldots \theta_{n}\right)$.

\subsection{Proof of the claim in Remark 1.5}

Here, we show that, in general, the polygon $\widehat{P}$ given by Theorem 1.4 is not circumscribed. We focus on the case of torsional rigidity and we argue by contradiction. For $n \geq 4$, we choose $\theta_{1}=\frac{\pi}{2}, \theta_{2}=\cdots=\theta_{n}=\frac{(2 n-5) \pi}{2(n-1)}$, and we denote by $\widehat{P}_{n}$ the corresponding polygon satisfying the overdetermined condition (1.17). Assume by contradiction that $\widehat{P}_{n}$ is circumscribed to a disc for every $n$. Up to a rescaling, we can assume that it circumscribes a disc of radius 1 . Moreover, we can set the position of the vertices so that, for every $n$, the vertex of the angle $\theta_{1}$ is fixed at the point $A_{1}:=(\sqrt{2}, 0)$. Then, as $n \rightarrow+\infty$, the sequence $\widehat{P}_{n}$ will collapse to the set $K$ which is the convex hull of the point $A_{1}$ and the disc of radius 1 . Accordingly, the torsion functions $u_{n}$ of $\widehat{P}_{n}$, extended by 0 on the complement of $\widehat{P}_{n}$, will converge strongly in $H^{1}\left(\mathbb{R}^{2}\right)$ to the torsion function $u$ of $K$. We are going to show that

$$
|\nabla u|=\text { constant } \quad \text { on }\left\{\mathrm{e}^{i \theta}: \frac{\pi}{3} \leq \theta \leq 2 \pi-\frac{\pi}{3}\right\} \subset \partial K .
$$

Since $K$ is not a disk, this contradicts the symmetry result for partially overdetermined problem proved in [12], Section 3.1 and achieves our proof. Let us prove (3.13). To that aim, let us fix a point $x=\mathrm{e}^{i \theta}, \frac{\pi}{3} \leq \theta \leq 2 \pi-\frac{\pi}{3}$ and a radius $\varepsilon \in\left(0, \sin \frac{\pi}{24}\right)$, and let us compute the limiting behavior, as $n \rightarrow+\infty$, of the sequence

$$
\int_{\partial \widehat{P}_{n} \cap B_{x, \varepsilon}}\left|\nabla u_{n}\right|^{2} \mathrm{~d} s
$$

On one hand, we have that

$$
\int_{\partial \widehat{P}_{n} \cap B_{x, \varepsilon}}\left|\nabla u_{n}\right|^{2} \mathrm{~d} s \rightarrow \int_{\Gamma \cap B_{x, \varepsilon}}|\nabla u|^{2} \mathrm{~d} s
$$

where $\Gamma:=\left\{\mathrm{e}^{i \theta}: \frac{\pi}{4} \leq \theta \leq 2 \pi-\frac{\pi}{4}\right\} \subset \partial K$. To see this observe that, for any $\varphi \in C_{0}^{\infty}\left(\mathbb{R}^{2}\right)$ such that supp $\varphi \cap \partial K \subset$ $\Gamma$, the sequence of measures $\varphi \cdot \mathcal{H}^{1}\left\llcorner\partial \widehat{P}_{n}\right.$ converge weakly $*$ to $\varphi \cdot \mathcal{H}^{1}\llcorner\Gamma$. Then, by using [7], Proposition 2.2 and the fact that the extension operators from $H^{1}\left(\widehat{P}_{n}\right)$ to $H^{1}\left(\mathbb{R}^{2}\right)$ are uniformly bounded, we get (possibly after extracting a subsequence)

$$
\int_{\partial \widehat{P}_{n}}\left|\nabla u_{n}\right|^{2} \varphi \mathrm{d} s \rightarrow \int_{\Gamma}|\nabla u|^{2} \varphi \mathrm{d} s
$$

which implies (3.14).

On the other hand, setting $C=\int_{\Gamma}|\nabla u|^{2} \mathrm{~d} s$, we have that

$$
\int_{\partial \widehat{P}_{n} \cap B_{x, \varepsilon}}\left|\nabla u_{n}\right|^{2} \mathrm{~d} s \rightarrow C \frac{\mathcal{H}^{1}\left(\Gamma \cap B_{x, \varepsilon}\right)}{\mathcal{H}^{1}(\Gamma)} .
$$


This follows from the overdeterminated condition on $\partial \widehat{P}_{n}$,

$$
\forall 1<i, j<n-1, \int_{A_{i}^{n} A_{i+1}^{n}}\left|\nabla u_{n}\right|^{2} \mathrm{~d} s=\int_{A_{j}^{n} A_{j+1}^{n}}\left|\nabla u_{n}\right|^{2} \mathrm{~d} s
$$

taking into account that the $L^{\infty}$-norms of $\nabla u_{n}$ are uniformly bounded. This last assertion holds true since the sequence of $H^{2}\left(\Omega_{n}\right)$-norms of $u_{n}$ is bounded, because $\widehat{P}_{n}$ are convex sets with bounded diameter and inradius bounded from below (see [15], identity 3.1.2.2).

Finally, combining (3.14) and (3.15), we obtain (3.13).

\subsection{Proof of Theorem 1.6}

It is enough to exploit the identities (1.19)-(1.20) holding by Corollary 2.5, and the convexity of the map $t \mapsto \cot (t / 2)$ over $(0, \pi)$.

Acknowledgements. DB was supported by the LabEx PERSYVAL-Lab GeoSpec (ANR-11-LABX-0025-01) and ANR SHAPO (ANR-18-CE40-0013).

\section{REFERENCES}

[1] C. Borell, Hitting probabilities of killed Brownian motion: a study on geometric regularity. Ann. Sci. École Norm. Sup. 17 (1984) 451-467.

[2] C. Borell, Greenian potentials and concavity. Math. Ann. 272 (1985) 155-160.

[3] H.J. Brascamp and E.H. Lieb, On extensions of the Brunn-Minkowski and Prékopa-Leindler theorems, including inequalities for $\log$ concave functions, and with an application to the diffusion equation. J. Funct. Anal. 22 (1976) 366-389.

[4] D. Bucur and I. Fragalà, A Faber-Krahn inequality for the Cheeger constant of N-gons. J. Geom. Anal. 26 (2016) 88-117.

[5] D. Bucur and I. Fragalà, Proof of the honeycomb asymptotics for optimal Cheeger clusters. Adv. Math. 350 (2019) 97-129.

[6] D. Bucur, I. Fragalà and J. Lamboley, Optimal convex shapes for concave functionals. ESAIM: COCV 18 (2012) 693-711.

[7] D. Bucur and M. Nahon, Stability and instability issues of the Weinstock inequality. Preprint arxiv 2004.07784 (2020).

[8] A. Colesanti, Brunn-Minkowski inequalities for variational functionals and related problems. Adv. Math. 194 (2005) 105-140.

[9] A. Colesanti and P. Cuoghi, The Brunn-Minkowski inequality for the $n$-dimensional logarithmic capacity of convex bodies. Potential Anal. 22 (2005) 289-304.

[10] A. Colesanti and M. Fimiani, The Minkowski problem for torsional rigidity. Indiana Univ. Math. J. 59 (2010) $1013-1039$.

[11] I. Fragalà, Symmetry results for overdetermined problems on convex domains via Brunn-Minkowski inequalities. J. Math. Pures Appl. 97 (2012) 55-65.

[12] I. Fragalà and F. Gazzola, Partially overdetermined elliptic boundary value problems. J. Differ. Equ. 245 (2008) $1299-1322$.

[13] I. Fragalà, F. Gazzola and J. Lamboley, Some sharp bounds for the p-torsion of convex domains. Geometric properties for parabolic and elliptic PDE's. In Vol. 2 of Springer INdAM Ser. (2013) 97-115.

[14] I. Fragalà and B. Velichkov, Serrin-type theorems on triangles. Proc. Am. Math. Soc. 147 (2019) 1615-1626.

[15] P. Grisvard, Elliptic problems in nonsmooth domains, in Vol. 69 Classics in Applied Mathematics. Reprint of the 1985 original [MR0775683], With a foreword by Susanne C. Brenner. Society for Industrial and Applied Mathematics (SIAM), Philadelphia, PA (2011).

[16] A. Henrot, Shape Optimization and Spectral Theory. De Gruyter (2017).

[17] D. Jerison, The direct method in the calculus of variations for convex bodies. Adv. Math. 122 (1996) $262-279$.

[18] D. Jerison, A Minkowski problem for electrostatic capacity. Acta Math. 176 (1996) 1-47.

[19] M. Jungen, A model of columnar jointing. Math. Models Methods Appl. Sci. 22 (2012) 1150006.

[20] C. Nitsch, An isoperimetric result for the fundamental frequency via domain derivative. Calc. Var. Partial Differ. Equ. 49 (2014) 323-335.

[21] G. Pólya and G. Szegö, Isoperimetric Inequalities in Mathematical Physics, Vol. 27 of Annals of Mathematics Studies. Princeton University Press, Princeton, N.J. (1951).

[22] R. Schneider, Convex bodies: the Brunn-Minkowski theory, in Vol. 151 Encyclopedia of Mathematics and its Applications, expanded ed.. Cambridge University Press, Cambridge (2014).

[23] A. Solynin, Isoperimetric inequalities for polygons and dissymetrization. Algebra Analiz 4 (1992) 210-234.

[24] A.Y. Solynin and V.A. Zalgaller, An isoperimetric inequality for logarithmic capacity of polygons. Ann. Math. 159 (2004) 277-303.

[25] J. Xiao, Exploiting log-capacity in convex geometry. Asian J. Math. 22 (2018) 953-978. 